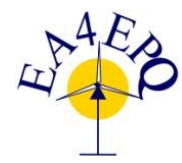

International Conference on Renewable Energies and Power Quality (ICREPQ'17)

Malaga (Spain), $4^{\text {th }}$ to $6^{\text {th }}$ April, 2017

Renewable Energy and Pourer Qualily. Fournal (RE\&PQJ)

ISSN 2172-038 X, No.15 April 2017

\title{
A Study on Optimal and Stable Strategy of Islanded Microgrid
}

\author{
Min-Hyeok Kang ${ }^{1}$, Ngoc-Thinh Quach ${ }^{1}$, Sang Heon Chae ${ }^{1}$, Dong Wan Kim², \\ Seung Hun $\mathrm{Ko}^{2}$, Eel-Hwan Kim ${ }^{1}$ and Seong-Bo $\mathrm{Oh}^{1}$ \\ ${ }^{1}$ Department of Electrical Engineering \\ Jeju National University \\ Jeju-si, Jeju Special Self-Governing Province, 63243, Republic of Korea \\ Phone/Fax number: +82 64 7543678, e-mail: minh0131@jejunu.ac.kr, ngocthinh@ctu.edu.vn, chae@jejunu.ac.kr, \\ ehkim@jejunu.ac.kr, sboh@jejunu.ac.kr \\ ${ }^{2}$ Research \& Development Center, Jeju Energy Corporation \\ Jeju-si, Jeju Special Self-Governing Province, 63243, Republic of Korea \\ E-mail: segi305@jejuenergy.or.kr, kosh5862@jejuenergy.or.kr
}

\begin{abstract}
This paper presents a study on optimal and stable operation strategy of an islanded microgrid. By considering the minimum power limit of the DG, the operating strategy is discussed in order to analyze the stability of the microgrid. Then, a method based on particle swarm optimization (PSO) algorithm is used to achieve multi-objective optimization such as the maximized renewable energy source penetration, the minimized $\mathrm{CO}_{2}$ emission, and the reliability of microgrid. The proposed operation strategy will be verified by using Matlab programming, PSCAD simulation program and measured data from power system on Chuja Island in 2015.
\end{abstract}

Key words: Diesel generator, Islanded Microgrid, Particle swarm optimization, Stability analysis, Renewable energy source penetration

\section{Introduction}

In islanded microgrid, the stability and reliability almost depend on energy storage system (ESS) and diesel generator (DG) because of the intermittent characteristics of the renewable energy sources (RESs) such as wind turbine generator (WTG) and photovoltaic system (PV). The design and optimization of the islanded microgrid have been researched by many authors over the world. The optimal sizing methods such as genetic algorithm (GA) and multi-objective evolutionary algorithm have been developed to determine the configuration of microgrid that can achieve the multi-objective optimization problem including the minimized life-cycle cost, the maximized renewable energy source penetration, the minimized pollutant emissions, and reliability. Although the GA is an efficient method to optimize the sizing of microgrids, the GA is not easy to code. Particle swarm optimization (PSO) algorithm is another method that is comparable to the GA, but it has a shorter calculation time, stable convergence characteristics, robustness, and easy coding implementation. Thus, the
PSO algorithm has become one of the favorite optimization methods, especially for optimization of the microgrid [1].

These optimal methods are used to determine the optimal sizing of each component in the new microgrid system. However, the application of these methods for the microgrid system that its sizing has been known in advance is not researched yet. Besides, a microgrid usually requires an energy management strategy to achieve a good compromission between stable and economic operation [2], but the mentioned methods only focus on the optimization objectives rather than the stable operation of microgrid. While the DG takes a few minutes for starting and warming up the diesel engine before connecting to power system, the power flow on the system is unbalanced. Moreover, the operation of DG under light-load conditions significantly increases the risk of engine failure, and can cause premature ageing of the DG [3]. Thus, this paper presents a study on the optimal and stable operation strategy of an islanded microgrid such as the power system on Chuja Island. In this case, the sizing of WTG, PV, ESS and DG have been decided by Jeju Special Self-Governing Province. Thus, a stable operation strategy of microgrid is analyzed in consideration of the minimum power limit of the DG. Then, the PSO algorithm is employed to achieve multiobjective optimization such as the maximized renewable energy source penetration, the minimized $\mathrm{CO}_{2}$ emission, and the reliability.

\section{Microgrid}

\subsection{Configuration}

At the present, the main power source on Chuja Island comes from the DGs. However, the Korean Government has a plan in order to reduce $\mathrm{CO}_{2}$ emission and increase 
the usage of RESs. Therefore, the power system on Chuja Island will be converted to an islanded microgrid which consists of WTG, PV, DG and ESS units as shown in Fig. 1. The size of each component on Chuja microgrid has been decided by the Jeju Special Self-Governing Provincial Government as shown in Table 1. The maximum electric load on Chuja Island in 2015 is 2232 $\mathrm{kW}$. Total nominal power of DGs is $5000 \mathrm{~kW}$.

The hourly wind velocity data on Chuja Island in 2015 is illustrated in Fig. 2. The wind velocity varies between $0 \mathrm{~m} / \mathrm{s}$ and $19.76 \mathrm{~m} / \mathrm{s}$. It is high at the beginning and the end of a year. The annual average wind velocity is about $5.17 \mathrm{~m} / \mathrm{s}$.

The hourly solar radiation data in 2015 is shown in Fig. 3. Generally, the solar radiation on Chuja Island is low during every period of a year. It is almost lower than $1.0 \mathrm{~kW} / \mathrm{m}^{2}$.

The hourly load demand in 2015 on Chuja Island is shown in Fig. 4. The maximum load is $2232 \mathrm{~kW}$ that occurs on summer. The minimum load is $1386 \mathrm{~kW}$ on fall.

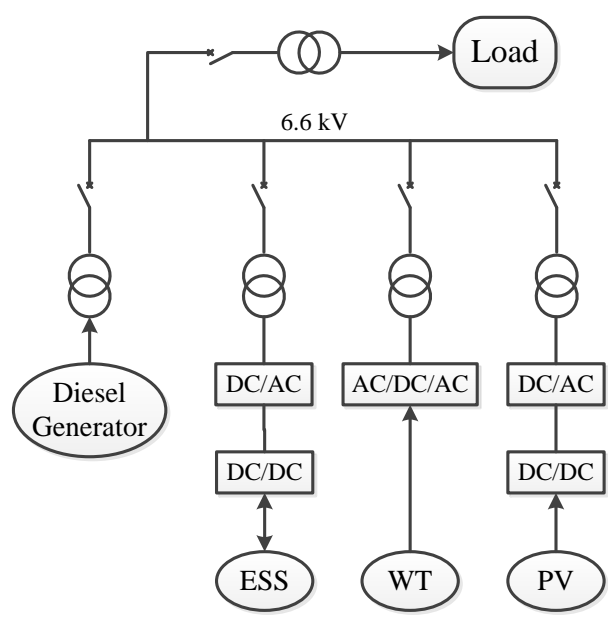

Fig. 1. Configuration of Chuja microgrid system

Table 1 . The sizing of microgrid

\begin{tabular}{|l|c|c|}
\hline \multicolumn{1}{|c|}{ Item } & Capacity & Quantity \\
\hline Wind turbine generator & $800 \mathrm{~kW}$ & 3 \\
\hline Photovoltaic system & $100 \mathrm{~kW}$ & 16 \\
\hline Battery storage system & $12 \mathrm{MWh}$ & 1 \\
\hline \multirow{2}{*}{ Diesel generator } & $1000 \mathrm{~kW}$ & 4 \\
\cline { 2 - 3 } & $500 \mathrm{~kW}$ & 2 \\
\hline Maximum electric load & $2232 \mathrm{~kW}$ & - \\
\hline
\end{tabular}



Fig. 2. Hourly wind velocity on Chuja Island in 2015

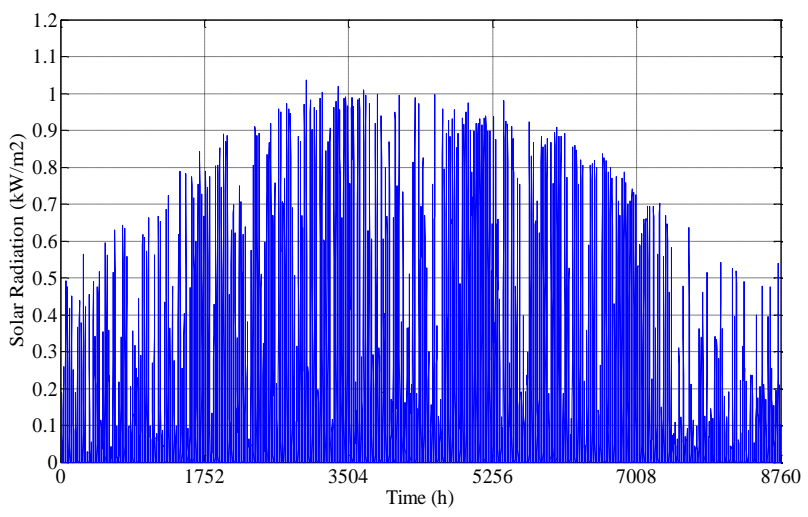

Fig. 3. Hourly solar radiation on Chuja Island in 2015

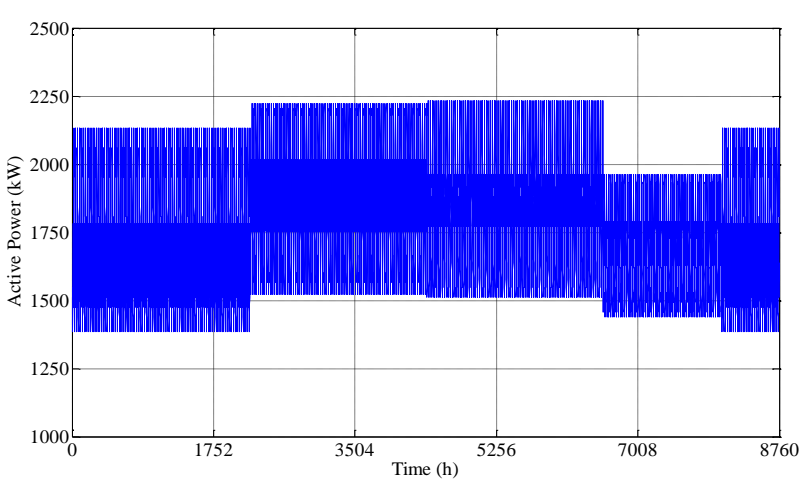

Fig. 4. Load profile on Chuja Island in 2015

\subsection{Operation}

In the microgrid, the operations of WTG and PV depend on the weather condition and control strategy. With the optimization objectives as mentioned before, the DG will be only turned on if the energy of RES and ESS is not enough for load demand. However, the starting time of DG prolongs a few minutes for warming up the diesel engine. During this duration, the DG must be operated under no load conditions. Therefore, the electric power on the system will be unbalanced during the starting time of DG. As a result, the grid voltage will be dropped out of the nominal range, and the microgrid system must be then turned off or at least the load demand must be curtailed. This is an unexpected situation. Besides, the operation of DG under light-load conditions significantly increases the risk of engine failure as well as premature ageing of the DG. To solve this problem, the DG will be operated based on a determined schedule that considers the minimum power limit of DG. The minimum output power of a DG is normally set between $30 \div 50 \%$ of rated power [2]. In this paper, the minimum power limit is set to $30 \%$ of rated power. The objective is to use the RES as a priority and to achieve stability of microgrid.

\section{Optimization}

\subsection{Particle swarm optimization}

In PSO algorithm, two best values determine each particle position such as a personal best (the best value of each particle) and global best (the best value of particle in the entire swarm) [4]. All particles are initiated randomly and evaluated to compute fitness function together with 
finding the personal best and global. After that, a loop starts to find an optimum solution. In the loop, each particle velocity is firstly updated by the personal and global bests, and each particle position is then updated by the current velocity. The loop is repeated until it meets a stopping criterion such as the number of iterations or predefined target fitness values as shown in Fig. 5.

The position of each particle is updated in the search space by

$$
x_{i}(t+1)=x_{i}(t)+v_{i}(t+1)
$$

where $x_{i}$ is the position of particle $i$ in the search space. $v_{i}$ denotes the velocity of particle $i$. $t$ is the discrete time steps.

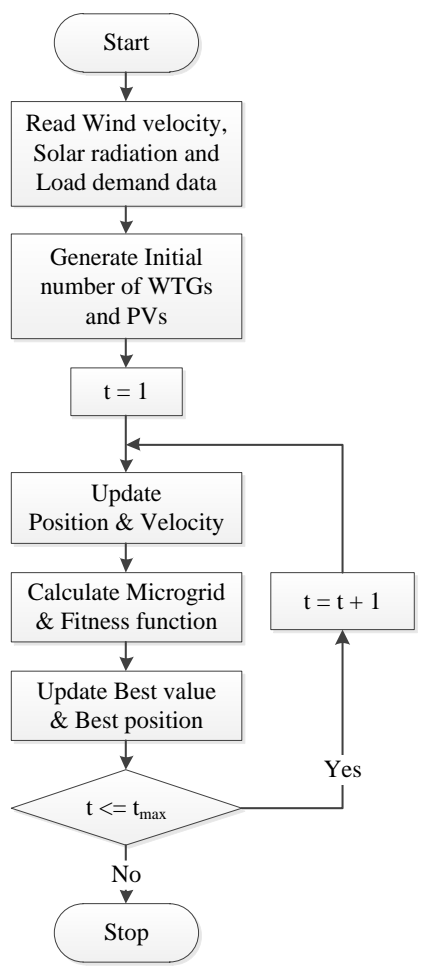

Fig. 5. Particle swarm optimization for Chuja microgrid

The velocity of particle $i$ is calculated as

$v_{i}(t+1)=\chi\left\{v_{i}(t)+\Phi_{1}\left[P_{\text {besti }}(t)-x_{i}(t)\right]+\Phi_{2}\left[G_{\text {best }}(t)-x_{i}(t)\right]\right\}$

where $G_{b e s t}$ is the global best position of the swarm. $P_{b e s t i}$ the personal best position of particle $i$. The constriction coefficient, $\chi$, is given by

$$
\begin{aligned}
& \chi=\frac{2}{\left|2-\Phi-\sqrt{\Phi^{2}-4 \Phi}\right|} \\
& \Phi=\Phi_{1}+\Phi_{2} \quad(\Phi>4) \\
& \Phi_{1}=c_{1} r_{1} \\
& \Phi_{2}=c_{2} r_{2}
\end{aligned}
$$

where $c_{1}$ and $c_{2}$ are the cognitive and social parameters. $r_{1}$ and $r_{2}$ denote the random numbers between 0 and 1 . It has been proposed that two acceleration constants should be $c_{1}=c_{2}=2$.

\subsection{Multi-objective optimization}

Optimization of Chuja microgrid is considered as a multi-objective optimization problem. A simple optimal method is to convert the multi-objective problem into a single objective problem. The optimization objectives for Chuja microgrid are the maximized renewable energy source penetration, the minimized $\mathrm{CO}_{2}$ emission, and the reliability of microgrid. Thus, the fitness function is expressed as

$$
\text { fitness }=\min \left(\mu_{1} \frac{1}{R e}+\mu_{2} \frac{w_{\mathrm{CO} 2}}{\left(w_{\mathrm{CO} 2}\right)_{\max }}+\mu_{3} \operatorname{LPSP}\right)
$$

with

$$
\begin{aligned}
& \sum_{i=1}^{3} \mu_{i}=1 \\
& \mathrm{Re}=\frac{\sum_{j=1}^{j=y}\left(E_{P V, j}+E_{W T, j}\right)}{\sum_{j=1}^{j=y}\left(E_{P V, j}+E_{W T, j}+E_{D G, j}\right)} \\
& w_{C O 2}=P_{\text {de_out }} . F_{C O 2} \\
& L P S P=\frac{\text { Totaltime }\left(P_{L}>P_{G e n}\right)}{T}
\end{aligned}
$$

where $\mu$ is the weighting factor of each objective. $w_{\mathrm{CO} 2}$ is the weight of $\mathrm{CO}_{2}$ emission per year. LPSP is the loss of power supply probability. $E_{P V, j}, E_{W T, j}$ and $E_{D G, j}$ are the total energy of PV, WTG and DG at year $j$ th, respectively. $P_{\text {de_out }}$ is the total output power of the DGs. $P_{L}$ is the total load demand in duration of $T . P_{G e n}$ is the total power generation of the microgrid system. 2.

The constraints of the PSO algorithm are given in Table

\section{Simulation results}

\subsection{Optimization objectives}

The results of running the PSO program for Chuja microgrid is shown in Table 3. It can be seen that all optimization objectives can be achieved at the same time. In this case, all of WTG and PV units are chosen. The capacity of three DG units are: $P_{D G 1}=500 \mathrm{~kW}, P_{D G 2}=$ $P_{D G 3}=1000 \mathrm{~kW}$. The output power of DG is regulated as soon as the RES and ESS cannot respond enough power to the load demand. The RES penetration is about $33.56 \%$ /year. In the study, the reliability of Chuja microgrid is always ensured in every case of operation because the total capacity of DGs is higher than that of the maximum load demand. This is expressed by $L P S P=0$.

Table 2. The constraints of PSO algorithm

\begin{tabular}{|l|c|c|}
\hline \multicolumn{1}{|c|}{ Item } & $\begin{array}{c}\text { Lower } \\
\text { bound }\end{array}$ & $\begin{array}{c}\text { Upper } \\
\text { bound }\end{array}$ \\
\hline Wind turbine generator & 0 & 3 \\
\hline Photovoltaic system & 0 & 16 \\
\hline Battery storage system & 0 & 1 \\
\hline Diesel generator $500 \mathrm{~kW}$ & 0 & 2 \\
\hline Diesel generator $1000 \mathrm{~kW}$ & 0 & 4 \\
\hline
\end{tabular}

Table 3. The result of running PSO program

\begin{tabular}{|c|c|c|c|c|c|c|}
\hline $\begin{array}{c}\text { WTG } \\
\text { (unit) }\end{array}$ & $\begin{array}{c}\text { PV } \\
\text { (unit) }\end{array}$ & $\begin{array}{c}\text { ESS } \\
\text { (unit) }\end{array}$ & $\begin{array}{c}\text { DG } \\
\text { (unit) }\end{array}$ & $\begin{array}{c}\text { Re } \\
(\%)\end{array}$ & $\begin{array}{c}\text { LPSP } \\
\text { (\%) }\end{array}$ & $\begin{array}{c}\text { CO}_{2} \\
\text { (ton/year) }\end{array}$ \\
\hline 3 & 16 & 1 & 3 & 33.56 & 0.0 & 7099.37 \\
\hline
\end{tabular}


The DG1 and DG2 are operated continuously to supply the electric power to the load demand. The $\mathrm{CO}_{2}$ emission is about 7099.37 ton/year. The operation of DG is shown in Fig. 6.

\subsection{Stability of Chuja microgrid}

To verify the stability of Chuja microgrid, the simulation is carried out by using the PSCAD/EMTDC simulation program. All DG units operate with the output power from $30 \%$ to $100 \%$ of rated power. However, the DG1 and DG2 are operated continuously during a year, excepting maintenance time, while the DG3 is controlled based on a determined schedule. The DG3 will be turned on if the reference powers of DG1 and DG2 reach to $95 \%$ of rated power. This work is to compensate for the starting time of DG3 and to reserve for the intermittent characteristics of the RES. Once the DGs are turned on, the load sharing of DGs are as follows.

$$
P_{D G i}=P_{D G}^{r e f} \frac{S_{D G i}}{S_{D G}} \quad i=1,2,3
$$

where $P_{D G}^{r e f}$ is total reference power of DG. $S_{D G i}$ denotes the apparent power of $i$ th DG. $S_{D G}$ is the rated apparent power of DG based on DGs in on-position.

The simulation results are shown in Fig. 7. In the simulation, it is assumed that the ESS is discharged fully $\left(C_{B}=C_{B m i n}\right)$. In other words, the Chuja microgrid is operating with the RES, DG and load. The load demand is expressed in Fig. 7(a). The minimum load is $1.3 \mathrm{MW}$, and the maximum load is $2.3 \mathrm{MW}$. The output powers of WTG and PV are shown in Fig. 7(b). The DGs can respond rapidly as soon as the load demand is changed as shown in Fig. 7(c). This is due to the DGs are always available in this case. It means that the DGs do not require the starting time. As a result, the grid voltage is stable during the simulation (Fig. 7(d)). The grid frequency is also stable as expressed in Fig. 7(e).

These results demonstrate the effectiveness of control strategy that the minimum power limit of DG is $30 \%$ of rated power. If the minimum power limit is higher than $30 \%$ of rated power, the efficiency of DG will be improved. However, the $\mathrm{CO}_{2}$ emission will be also increased at the same time. This is an unexpected situation. Thus, the range of $30 \% \div 100 \%$ of rated power is quite reasonable for the operation of DG.

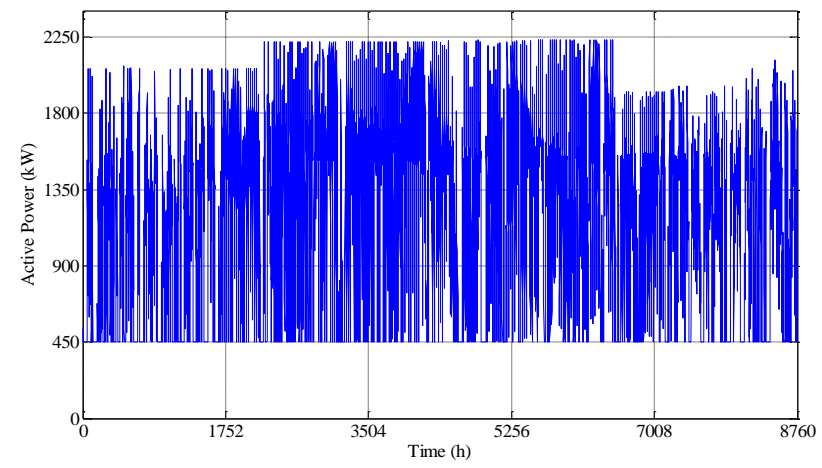

Fig. 6. The operation of diesel generator

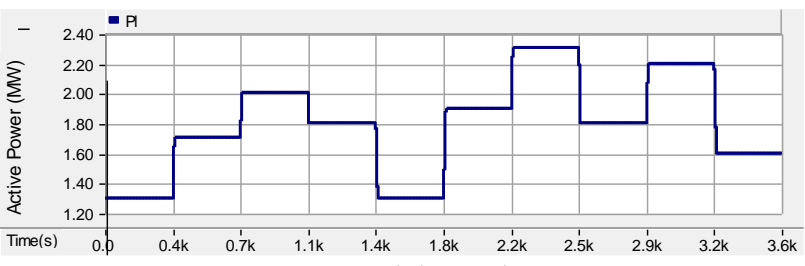

(a) Load demand

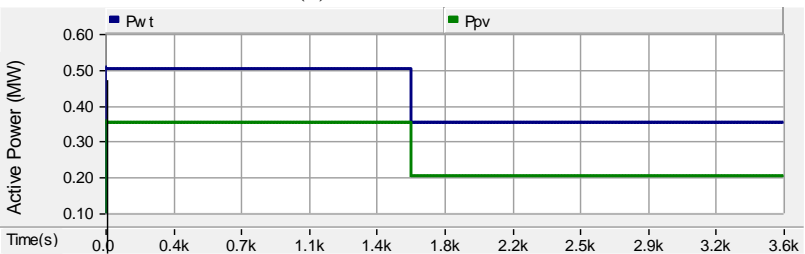

(b) Output power of WTG and PV

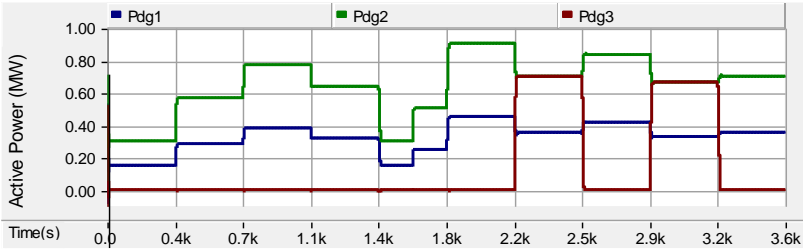

(c) Output power of DG

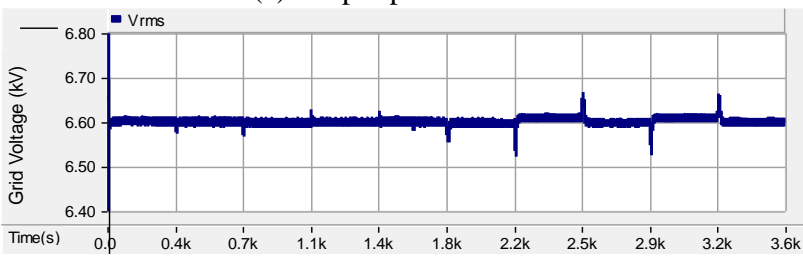

(d) Grid voltage

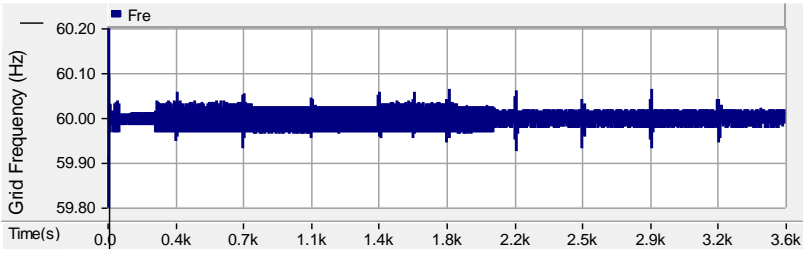

(e) Grid frequency

Fig. 7. The operation of Chuja microgrid

\section{Conclusion}

This paper has presented a study on the optimal and stable operation strategy of an islanded microgrid such as Chuja microgrid. By using the PSO algorithm, the optimization objectives of Chuja microgrid such as the maximized renewable energy source penetration, the minimized $\mathrm{CO}_{2}$ emission, and the reliability of microgrid have been achieved. The reliability of the microgrid is always ensured during the operating time $(L P S P=0)$. The voltage and frequency of the microgrid are stable. This demonstrates that the stability of Chuja microgrid can achieve at the same time with the optimization objectives when the minimum power limit of DG is about $30 \%$ rated power. Besides, the lifetime of the DGs is also improved.

\section{References}

[1] Hanieh Borhanazad, Saad Mekhilef, Velaapa Gounder Ganapathy, Mostafa Modiri-Delshad and Ali Mirtaheri, "Optimization of Micro-Grid System Using MOPSO," Renewable Emnergy, vol. 71, pp. 295-306, Nov. 2014 
[2] Farid Katiraei and Chad Abbey, "Diesel Plant Sizing and Performance Analysis of a Remote Wind-Diesel Microgrid," 2007 IEEE Power Engineering Society General Meeting, Florida, USA, pp. 1-8, June 2007.

[3] Makbul A. M. Ramli, Ayong Hiendro, and Yusuf A. AlTurki, "Techno-Economic Energy Analysis of Wind/Solar Hybrid System: Case Study for Western Coastal Area of Saudi Aribia," Renewable Energy, vol. 91, pp. 374-385, Jun. 2016.

[4] J. Kennedy and R. Eberhart, "Particle Swarm Optimization," in IEEE Proceedings of the International Conference on Neural Networks, Perth, Australia, pp. 1942-1948, 1995. 